\title{
On the potential effect of circadian rhythms of cardiac troponins on the diagnosis of acute myocardial infarction
}

\author{
Aleksey Michailovich Chaulin ${ }^{1, \star}$, Dmitry V. Duplyakov ${ }^{1}$
}

\begin{abstract}
${ }^{1}$ Department of Cardiology and Cardiovascular Surgery, Samara State Medical University, Chapaevskaya Str. 89, 443099 Samara, Russia
\end{abstract}

\section{*Correspondence}

alekseymichailovich22976@gmail.com (Aleksey Michailovich Chaulin)

\begin{abstract}
Methods for the determination of cardiac troponins have been significantly improved, facilitating further advancement of the therapeutic and diagnostic process of the cardiovascular diseases case management. In particular, acceleration of the acute myocardial infarction testing (early diagnostic algorithms: 0-1 h, 0-3 h) enabled earlier detection and choice of the optimal treatment tactics. At the same time, with the increased sensitivity in the laboratory test results interpretation, there emerged a necessity to take into account some additional factors that may affect the concentration of cardiac troponins in blood serum. Several recent studies have reported the existence of circadian rhythms in cardiac troponins. This article aims to discuss the possible mechanisms of how circadian rhythms of cardiac troponins develop and their influence on the diagnosis of acute myocardial infarction.
\end{abstract}

\section{Keywords}

Cardiac troponins; Cardiovascular diseases; Acute myocardial infarction; Circadian rhythms; High-sensitive analyses

\section{Introduction}

To date, cardiospecific troponin isoforms determined by highsensitive methods (hs-cTnI and hs-cTnT) are the most preferred biomarkers in the detection of acute myocardial infarction (AMI) $[1,2]$. Nevertheless, together with the sensitization (detecting ability) of these methods, a slight decrease in the specificity of hs-cTnI and hs-cTnT took place, which changed the concept of their diagnostic and prognostic value in terms of cardiovascular (CVD) and other diseases. Previously thought that cardiac troponins are strictly intracellular molecules, and their presence in biological fluids was considered as an important AMI pathological sign (diagnostic criterion), indicating cardiomyocyte death [3]. However, with the advent of new highly sensitive determination methods (hs-cTnI and hscTnT), these views have changed. So it was shown that modern highly sensitive methods of immunoassay could determine cardiac troponins for absolutely all healthy individuals, not only in blood [4] but also in other biological fluids, such as urine [5], saliva [6-8].

A decrease in specificity is expressed in the fact that hscTnI and hs-cTnT can increase under some other pathological conditions, that in a way affect the cardiovascular system (CVS) (sepsis, myocarditis, chronic kidney disease (CKD), use of cardiotoxic drugs (for example, chemotherapeutic agents) and others) (Fig. 1) [9-12], basically other than ischemia of the striated heart muscle, which requires clinicians to be more careful in interpreting overestimated and borderline results. Thus, hs-cTnI and hs-cTnT should be generally considered specific biomarkers of myocardial injury, but not for any particular type, including the cardiac muscle ischemic necrosis. Moreover, the levels of hs-cTnI and hs-cTnT can increase several times even in obviously harmless conditions such as physical exertion and psychoemotional stress [13, 14]. Therefore, the final diagnosis of AMI, in no case should be based only on the results of laboratory tests.

However the decrease in specificity is not such a profound drawback as evidenced by the rapidly increasing demand for modern highly sensitive research methods. According to a recent study, many healthcare facilities have almost completely switched to the new methods (hs-cTnI and hs-cTnT) in routine clinical practice for AMI diagnosis [15].

Today there are a large number of manufacturers of highsensitive test systems, different in analytical (quality) characteristics. For practical use, only those high-sensitive immunoassays are suitable that meet two key criteria: 1) the coefficient of variation (CV\%), intended to estimate serial measurements scatter in one sample, should not exceed $10 \%$ $(\mathrm{CV} \% \leq 10 \%)$ when troponin concentration is within the 99th percentile (accepted as the upper limit of the norm); 2) the level of troponins in the range from the limit of detection (LoD) to the level of the 99th percentile should be determined in more than $50 \%$ of the examined individuals from two populations, including at least 300 women and men, respectively. Given these requirements, the accurate determination of low troponin levels, comprising only a few $\mathrm{ng} / \mathrm{L}$ in healthy individuals, is challenging [16].

Special attention should be paid to the possibility of using 
TA B L E 1. hs-cTnI levels used for CVD risk stratification [16]

\begin{tabular}{lcc} 
CVD developing risk & hs-cTnI concentration in women & hs-cTnI concentration in men \\
\hline Low & $<4 \mathrm{ng} / \mathrm{L}$ & $<6 \mathrm{ng} / \mathrm{L}$ \\
Moderate & $4-10 \mathrm{ng} / \mathrm{L}$ & $6-12 \mathrm{ng} / \mathrm{L}$ \\
High & $>10 \mathrm{ng} / \mathrm{L}$ & $>12 \mathrm{ng} / \mathrm{L}$ \\
\hline
\end{tabular}

hs-cTnI and hs-cTnT to assess the CVD development risk in a healthy population. Thus, according to a large study involving 9005 people, exceeding hs-cTnI levels ( $>10 \mathrm{ng} / \mathrm{L}$ for women and $>12 \mathrm{ng} / \mathrm{L}$ for men) were associated with a high risk of CVD developing in the long term, while at lower levels of hs-cTnI, the risk of CVD developing significantly decreased (Table 1) $[16,17]$.

The use of highly sensitive determination methods encourages consideration of various factors that can affect the research result. The latter, as shown by recent studies, include circadian rhythms, the effect, and the possible underlying mechanisms of which are discussed in this review.

\section{Circadian rhythms of cardiac troponins}

Circadian (circadian or diurnal) rhythms are understood as cyclical fluctuations in the intensity of various kinds of biological processes and body functions associated with the alternation of day and night. Along with the biorhythms that determine the performance of the organism functions (for example, the rhythm of sleep/wakefulness, blood pressure (BP) fluctuations, heart rate (HR), etc.), there exist so-called regulatory biorhythms, in particular, circadian rhythms of hormone secretion $[18,19]$. To date, it has been established that most hormones (metabolism regulators) and metabolites, affected by the aforesaid regulators, have diurnal concentration variations, which should be considered in a study (selecting the time of biomaterial sampling) and interpreting the results.

With the advent of high-sensitive methods for cardiac troponin determination, it was noted that the concentration of hs-cTnT in the blood can also express a circadian rhythm [20-22]. K Aakre and colleagues studied weekly and 90minute biological variations in hs-cTnT (Roche Diagnostics) and hs-cTnI (Abbott Diagnostics) levels in CKD hemodialysisdependent $(\mathrm{n}=19)$ and healthy patients $(\mathrm{n}=20)$. Blood sampling from chronic kidney disease patients took place when no hemodialysis was performed. It was found that over a 6 hour period (from the moment of the first blood draw at 8:30 am to the last at 2:30 pm), the hs-cTnT and hs-cTnI concentrations gradually decreased [21].

In a clinical study, Klinkenberg et al. conducted a standardized assessment of intra-day, diurnal, and weekly biological changes in hs-cTnT levels (Roche Diagnostics) [22]. To examine these circadian variations in hs-cTnT concentration, the authors conducted 2 separate studies. The target of the research included type 2 diabetes mellitus patients without acute CVD. In the first study, blood sampling $(\mathrm{n}=23)$ was performed through a venous catheter installed in the anterior ulnar veins 5 minutes before each standard meal (breakfast-8-30, lunch-1230, dinner-17-00), 90 and 150 minutes after each meal. Thus, each patient delivered 9 bioassays [22].

In a second study, to assess the diurnal variation, the researchers performed hourly hs-cTnT measurements in 7 patients, starting at 8-30 am (before breakfast) and completing at 9-30 am the next day. Thus, 25 measurements were made for each patient during this period of time. Patients followed a standard daily routine (diet and sleep), and night blood draws were performed without sleep disturbance. According to the results obtained, the researchers found that hs-cTnT concentrations do not vary by chance, but express certain tendencies: the maximum concentration was observed in the morning (8-30), while he hs-cTnT level decreased during the day, and in the evening (19-30) a smooth increase resumed. Thus, the concentration of hs-cTnT at time 8-30 a.m. significantly exceeded the evening values: median $=11.8 \mathrm{ng} / \mathrm{L}$ (interquartile interval: 9.3 to $15.2 \mathrm{ng} / \mathrm{L}$ ) versus median $=8.6$ ng/L [interquartile interval: 6.7 to $10.8 \mathrm{ng} / \mathrm{L}$ ]; $P<0.001$ ), respectively. It is estimated that, on average, the hs-cTnT level decreased by $24 \%$ over this period of time [22]. It is noteworthy that a similar pattern in the form of a gradual decrease during the day was previously noted for another marker (cardiac enzyme), namely, creatine kinase [23].

Another work of L. Klinkenberg and colleagues was devoted to circadian rhythms hs-cTnT and hs-cTnI. The authors found hs-cTnT circadian rhythms characterized by higher concentrations in the morning (about $16.2 \mathrm{ng} / \mathrm{L}$ at $8-30 \mathrm{am}$ ) and lower levels in the evening (about 12.1 at 7-30 pm). Besides, individuals with the highest hs-cTnT concentrations demonstrated the largest amplitude of daily fluctuations, as evidenced by the close correlation between the hs-cTnT concentration in the morning and the range of individual changes in hscTnT values over a 25 -hour period $(\mathrm{r}=0.70 ; P<0.001)$ [24]. However, unlike hs-cTnT, hs-cTnI showed no typical statistically significant differences in values during the day.

The results obtained by L. Klinkenberg are in good agreement with the data of N. Linden et al., who described an interesting clinical case [25]. Significant circadian variations in hs-cTnT concentration were found in an elderly patient with severe chronic kidney disease (estimated glomerular filtration rate $=14 \mathrm{~mL} / \mathrm{min} / 1.73 \mathrm{~m}^{2}$ ). Determination of hs-cTnT and hs-cTnI in the blood was carried out hourly for 25 hours (from 8-30 am the previous day and until 8-30 am the next day). The initial concentration of hs-cTnT in CKD patient $(147.7 \mathrm{ng} / \mathrm{L})$, in contrast to the initial concentration of hs-cTnI $(10.3 \mathrm{ng} / \mathrm{L})$, was more than 10 times higher than the values of the 99th-percentile (14 ng/L). The levels of hs-cTnT, in contrast to hs-cTnI, were "chronically" increased throughout the measurement period (several times exceeding the values of the 99th-percentile) (Fig. 2). The hs-cTnT concentrations in CKD a patient showed a circadian rhythm corresponding to the cosine model according to the cosine rhythmometry method 


\section{CARDIAC-RELATED CAUSES}

Inflammatory heart disease (myocarditis, endo/peri-myocarditis)

Cardiomyopathy (all types)

$\square$ Takotsubo cardiomyopathy

Cardiac insufficiency

Cardiotoxic compounds and medications (chemotherapy, sympathomimetics, cocaine, methamphetamine, etc.)

$\square$ Iatrogenic and surgical procedures that damage the heart (hemodialysis, PCI, catheter ablation, lefibrillator discharge, etc.)

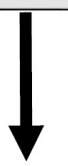

\section{CONSTITUTIONAL CONDITIONS}

$\square$ Prolonged and/or excessive physical activity

- Psychoemotional stress

- Kidney disease

Sepsis

- Pulmonary embolism (PATE)

- Neurological diseases (ischemic and hemorrhagic stroke, subarachnoid hemorrhage)

- Skeletal muscle diseases (idiopathic inflammatory myopathies, rhabdomyolysis, etc.)

Hypotension (shock) and hypoxia (severe respiratory distress, severe anemia, etc.)

\section{1 \\ ANALYTICAL AND \\ PREANALYTICAL \\ FACTORS}

$\square$ Heterophilic antibodies

Rheumatoid factor

$\square$ Cross-reaction with skeletal muscle troponins

$\square$ Hemolysis, lipemia, fibrin clots

Alkaline phosphatase

- Analyzer malfunction

F I G U R E 1. Ultimate reasons for hs-cTnI и hs-cTnT increase, apart from AMI according to [8, 9] amended and revised.

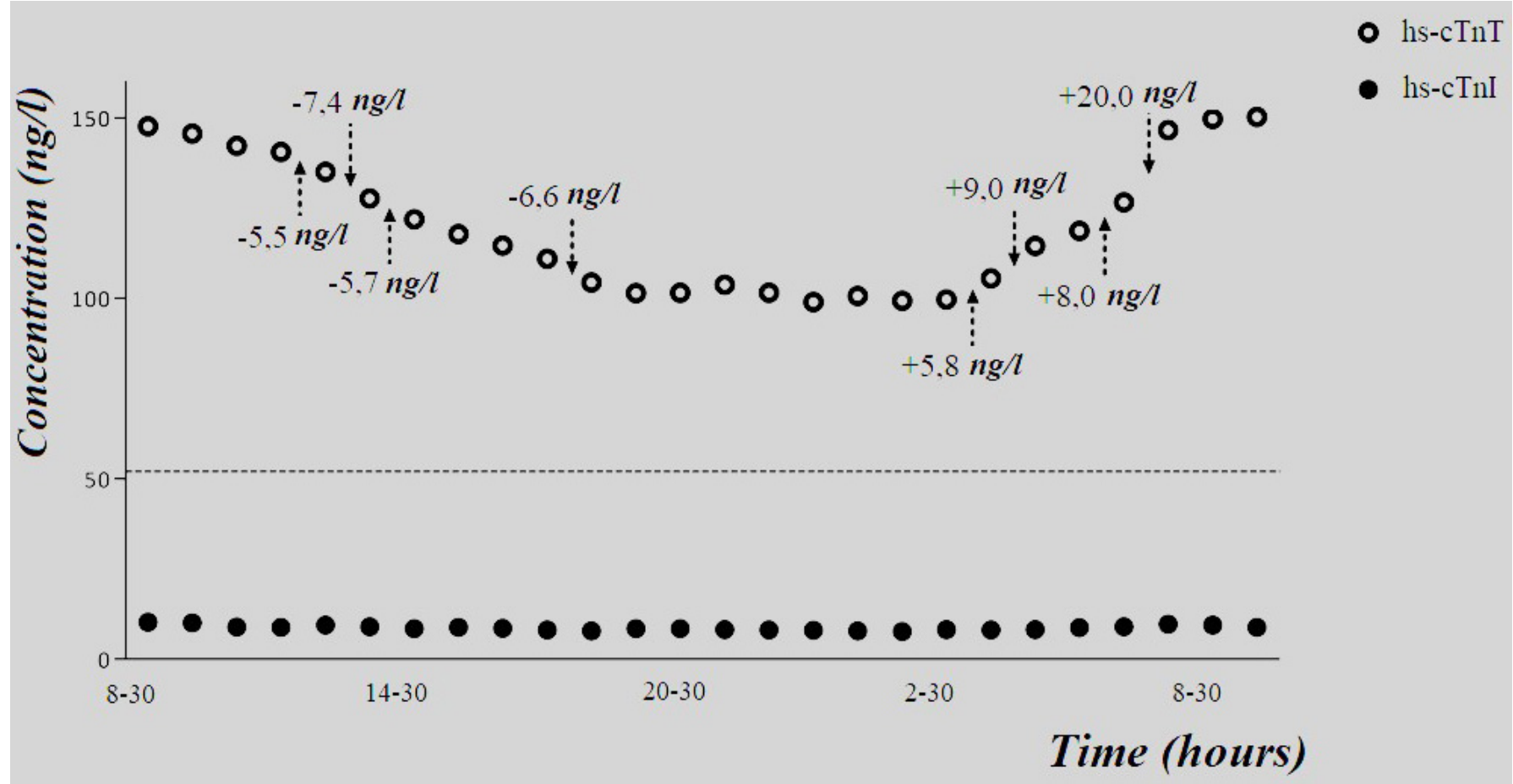

F I G U R E 2. Circadian fluctuations in concentration of hs-cTnT and hs-cTnI, as per [25] amended and revised. Notes. The hs-cTnT concentration curve shows one-hour changes that exceed the 1-hour delta-changes according to the recommended ESC 1-hour algorithms. The dashed line marks the hs-cTnT level (52 ng/L) for the quick exclusion of AMI without ST-segment elevation following the current diagnostic algorithms recommended by ESC [2].

developed by W. Nelson et al. [26] $(\mathrm{R} 2=0.90)$ with $50.9 \mathrm{ng} / \mathrm{L}$ being a maximum change in concentration within 24 hours. In contrast, hs-cTnI levels remained practically unchanged over time with a maximum difference of just $2.6 \mathrm{ng} / \mathrm{L}$ over the course of a day and no visual match to the cosine model. Notably, the concentration of hs-cTnT exceeded not only the 99th-percentile baseline level, which is, incidentally, typical for most chronic kidney disease patients [27] but also deltachanges used in modern 1- and 3-hour algorithms for AMI diagnostics, developed by experts from European Society of Cardiology (ESC) [2, 28, 29]. According to these algorithms, if the level of hs-cTnT (Roche diagnostics) is $52 \mathrm{ng} / \mathrm{L}$ upon admission to a hospital with suspected AMI, then AMI is diagnosed and the required amount of treatment is performed 
according to the protocol without additional serial measurements. Also, the 1-hour delta changes $(>5 \mathrm{ng} / \mathrm{L})$ used in the diagnosis of AMI by ESC for this test system were exceeded in the mentioned patient eight times a day (especially around noon and early morning). Thus, in contrast to the results of studies by L. Klinkenberg et al. [22, 24], the data of this study indicate that the natural circadian rhythms of hs-cTnT can directly affect the accuracy of AMI diagnosis when the rapid diagnostic algorithms recommended by the ESC are applied [23].

Despite several consistent results regarding the existence of hs-cTnT circadian rhythms, hs-cTnI determination methods do not record the same. Thus, K. Wildi et al., studying the levels of hs-cTnI, used three analysis methods (Abbott Architect, Siemens Ultra, Beckman Accu) and did not reveal significant differences in morning and evening troponin I level in the same patients [30]. However, given the huge variety of existing methods for determining hs-cTnI, the presence of circadian changes in hs-cTnI cannot be completely ruled out in the study of blood serum by other immunoassays.

It is also worth noting an important drawback of the clinical studies conducted on the study of hs-cTnT circadian rhythms: a small sample of examined individuals, which limits the output clinical and diagnostic value. An important factor that seems to restrict the conduct of large studies on the hs-cTnT and hs-cTnI circadian rhythms is their cost. So, for example, to assess circadian rhythms (the hourly study of troponins) in 100 subjects, it is necessary to conduct about 2.5 thousand analyzes. Nevertheless, such large studies are urgently needed for reliable assessment of the circadian rhythm impact on the accuracy of early CVD detection (screening) and algorithms for the AMI diagnosis. The second important restriction in assessing the significance of the cardiac troponins circadian rhythms is a large number of manufacturers of high-sensitive test systems [31], possessing different analytical characteristics and, probably, the effect of circadian rhythms on the diagnostic value will differ depending on test system.

\section{Possible mechanisms of the formation of circadian fluctuations in the concentration of cardiac troponins}

Elucidation of the causes and mechanisms underlying the formation of circadian fluctuations in the levels of cardiac troponins is extremely important since it allows to definitively establishing whether it is necessary to take into account circadian variations in the concentrations of these cardiomarkers in routine clinical practice.

A relation to circadian changes in the physiology of the CVS and the hemostatic system is proposed as a possible explanation for the increased levels [32-37]. For example, Soviet and foreign scientists more than 50 years ago established that soon after awakening healthy patients demonstrate body temperature and blood pressure rise during the day, while in the evening when preparing for sleep a decrease in these parameters is contrarily noted $[32,33]$. Later, the circadian rhythm was also described for several other indicators of CVS and the hemostatic system: heart rate (HR), the activity of the sympathetic nervous system, vascular resistance, renin-angiotensin- aldosterone system, coagulation hemostasis enzymes activity, platelet aggregation, and activity of the fibrinolysis system [32-37]. All the above components exhibit peak activity in the morning hours, which is necessary for a normal healthy individual to maintain an optimal wakefulness period. It is noteworthy that the peak activity of these parameters also coincides with the maximum concentration of hs-cTnT during the day, which may indicate their possible effect on the release of cardiac troponin molecules from myocardial cells.

Studies analyzing the effect of circadian rhythms on the severity and prognosis of AMI are quite interesting [38-42]. According to some data, circadian fluctuations of the sympathoadrenal system, clotting, and the endocrine system activity may coincide with circadian variations in the size of the necrotic focus in AMI, which also indirectly indicates the importance of these systems activity to the regulation of the degree of myocardial damage and the level of cardiomarkers in the blood serum. Thus, according to a retrospective study, the infarction size depends on the time of day: the largest size of the AMI zone is observed in patients admitted in the morning [38]. In another study by Arroyo Úcar et al. [39], patients with AMI symptoms were divided into two groups depending on the time of admission: 1) 0-12 hours; 2) 12-24 hours. Patients of the first ("morning") group had a significantly higher concentration of cardiac troponin I compared with the second group $(70.85 \pm 16.38$ versus $60.90 \pm 22.92 \mathrm{ng} / \mathrm{mL}$, respectively, $P=0.003$ ), which indicates more severe AMI in the first group. And according to multivariate analysis, the onset of AMI in the time interval 0-12 hours turned out to be an independent predictor of the size of the infarction zone (odds ratio $1.133,95 \%$ confidence interval $1.012-1.267, P=0.01$ ) [39].

Yu. Tsareva and colleagues reported on the existence of circadian rhythms of thyroid hormones and cortisol (in most patients morning concentrations are higher than in the evening hours), which is associated with a more severe clinical picture of CVD [19]. Thyroid hormones are known to potentiate the effect of catecholamines on the heart, increasing heart rate. An increase in heart rate, in turn, leads to a shortening of the myocardium relaxation period (diastole), when the myocardium blood filling happens. Reportedly, the heart rate is associated with the cardiac troponins level. Some researchers even reported the presence of a close correlation between heart rate and troponin $\mathrm{T}$ concentration [43]. Considering the work of Yu. Tsareva et al. (which states that cortisol concentrations are higher in the morning) [19] and the study by A Lazzarino et al. (where cortisol values are shown to be associated with hs-cTnT levels) [13], it can be assumed that cortisol is also one of the important factors contributing to circadian (diurnal) differences in hs-cTnT concentration in patients.

The reason for the differences in the existence of circadian rhythms hs-cTnT and hs-cTnI remains unclear. Although, it should be once again emphasized that due to the wide variety of methods for analyzing troponin I, the absence of circadian rhythms in the study by several methods (Abbott Architect, Siemens Ultra, Beckman Accu) $[24,25,30]$ is not a reason to believe that the same will be typical for other immunoassays. More research is needed to finally shed light on the existence of clinically significant hs-cTnT and hs-cTnI circadian rhythms. 


\section{Conclusions}

Thus, according to several recent studies, rhythmic daily fluctuations in concentration are peculiar of hs-cTnT: maximum values are observed in the morning, after which they gradually decrease during the day, and then again there is a gradual increase to morning values. According to some data, hscTnT circadian rhythms can have a significant impact on the accuracy of CVD early detection (screening) and even the diagnosis of AMI. Additional studies using larger samples are essential to more accurately assess the impact of hs-cTnT circadian rhythms on rapid algorithms for the AMI diagnosis. These studies will make it possible to finally decide whether, in addition to gender features of the 99th-percentile, it is worth introducing circadian features of the 99th-percentile and delta values of hs-cTnT depending on the time of patient admission.

The mechanisms underlying the formation of circadian fluctuations in the concentration of cardiac troponins are complicated in nature, including changes in the functioning of the cardiovascular system (increased heart rate, vascular resistance, blood pressure), hemostasis system (coagulation hemostasis enzymes activity, platelet aggregation, and fibrinolysis activity), as well as endocrine system (reninangiotensin-aldosterone system, the activity of the thyroid gland and adrenal cortex).

\section{AUTHOR CONTRIBUTIONS}

Aleksey M. Chaulin and Dmitry V. Duplyakov designed the study. Aleksey M. Chaulin and Dmitry V. Duplyakov collected the data. Aleksey M. Chaulin analyzed the results and drafted the manuscript. Dmitry V. Duplyakov edited the manuscript.

\section{ACKNOWLEDGMENT}

Thanks to all the peer reviewers for their opinions and suggestions.

\section{FUNDING}

This study was not funded.

\section{CONFLICT OF INTEREST}

The authors declare that there is no conflict of interest regarding the publication of this article.

\section{DATA AVAILABILITY}

The data used to support the findings of this study are available from the corresponding author upon request.

\section{REFERENCES}

[1] Thygesen K, Alpert JS, Jaffe AS, Chaitman BR, Bax JJ, Morrow DA, et $a l$. Fourth universal definition of myocardial infarction (2018). European Heart Journal. 2018; 40: 237-269.

[2] Roffi M, Patrono C, Collet J, Mueller C, Valgimigli M, Andreotti F, et al. 2015 ESC Guidelines for the management of acute coronary syndromes in patients presenting without persistent ST-segment elevation: task force for the management of acute coronary syndromes in patients presenting without persistent ST-segment elevation of the European Society of Cardiology (ESC). European Heart Journal. 2016; 37: 267-315.

[3] Myocardial infarction redefined-A consensus document of The Joint European Society of Cardiology/American College of Cardiology Committee for the Redefinition of Myocardial Infarction. European Heart Journal. 2000; 21: 1502-1513.

[4] Garcia-Osuna A, Gaze D, Grau-Agramunt M, Morris T, Telha C, Bartolome A, et al. Ultrasensitive quantification of cardiac troponin I by a Single Molecule Counting method: analytical validation and biological features. Clinica Chimica Acta. 2018; 486: 224-231.

[5] Pervan P, Svaguša T, Prkacin I, Savuk A, Bakos M, Perkov S. Urine high sensitive Troponin I measuring in patients with hypertension. Signa Vitae. 2017; 62-64.

[6] Chaulin AM, Karslyan LS, Grigorieva EV, Nurbaltaeva DA, Duplyakov DV. Clinical and diagnostic value of cardiac markers in human biological fluids. Kardiologiia. 2019; 59: 66-75. (In Russian)

[7] Mirzaii-Dizgah I, Riahi E. Salivary high-sensitivity cardiac troponin T levels in patients with acute myocardial infarction. Oral Diseases. 2013; 19: 180-184.

[8] Chaulin AM, Duplyakova PD, Bikbaeva GR, Tukhbatova AA, Grigorieva EV, Duplyakov DV. Concentration of high-sensitivity cardiac troponin I in the oral fluid in patients with acute myocardial infarction: a pilot study. Russian Journal of Cardiology. 2020; 25: 3814. (In Russian)

[9] Chaulin AM, Duplyakov DV. Increased cardiac troponins, not associated with acute coronary syndrome. Part 1 . Kardiologiya: novosti, mneniya, obuchenie [Cardiology: News, Opinions, Training]. 2019; 7: 13-23. (In Russian)

[10] Chaulin AM, Duplyakov DV. Increased cardiac troponins, not associated with acute coronary syndrome. Part 2. Kardiologiya: novosti, mneniya, obuchenie [Cardiology: News, Opinions, Training]. 2019; 7: 24-35. (In Russian)

[11] Chaulin AM, Duplyakov DV. Increased natriuretic peptides not associated with heart failure. Russian Journal of Cardiology. 2020; 25: 4140. (In Russian)

[12] Chaulin AM, Abashina OE, Duplyakov DV. Pathophysiological mechanisms of cardiotoxicity in chemotherapeutic agents. Russian Open Medical Journal 2020; 9: e0305.

[13] Lazzarino AI, Hamer M, Gaze D, Collinson P, Steptoe A. The association between cortisol response to mental stress and high-sensitivity cardiac troponin $\mathrm{T}$ plasma concentration in healthy adults. Journal of the American College of Cardiology. 2013; 62: 1694-1701.

[14] Richardson AJ, Leckie T, Watkins ER, Fitzpatrick D, Galloway R, Grimaldi $\mathrm{R}$, et al. Post marathon cardiac troponin $\mathrm{T}$ is associated with relative exercise intensity. Journal of Science and Medicine in Sport. 2018; 21: 880-884.

[15] Anand A, Shah ASV, Beshiri A, Jaffe AS, Mills NL. Global adoption of high-sensitivity cardiac troponins and the universal definition of myocardial infarction. Clinical Chemistry. 2019; 65: 484-489.

[16] Clerico A, Padoan A, Zaninotto M, Passino C, Plebani M. Clinical relevance of biological variation of cardiac troponins. Clinical Chemistry and Laboratory Medicine. 2020. (in press)

[17] Sigurdardottir FD, Lyngbakken MN, Holmen OL, Dalen H, Hveem K, Røsjø $\mathrm{H}$, et al. Relative prognostic value of cardiac troponin I and creactive protein in the general population (from the nord-trøndelag health [HUNT] study). American Journal of Cardiology. 2018; 121: 949-955.

[18] Mazzoccoli G, Carughi S, Sperandeo M, Pazienza V, Giuliani F, Tarquini R. Neuro-endocrine correlations of hypothalamic-pituitary-thyroid axis in healthy humans. Journal of Biological Regulators and Homeostatic Agents. 2011; 25: 249-257.

[19] Tsareva YO, Mayskova EA, Fedotov EA, Shvarts YG. Circadian rhythms of thyroid hormones in patients with ischemic heart disease, arterial hypertension, and atrial fibrillation. Kardiologiia. 2019; 59: 23-29.

[20] Wu AHB, Lu QA, Todd J, Moecks J, Wians F. Short- and long-term biological variation in cardiac troponin I measured with a high-sensitivity assay: implications for clinical practice. Clinical Chemistry. 2009; 55: 52-58.

[21] Aakre KM, Røraas T, Petersen PH, Svarstad E, Sellevoll H, Skadberg, et al. Weekly and 90-minute biological variations in cardiac troponin $\mathrm{T}$ and 
cardiac troponin I in hemodialysis patients and healthy controls. Clinical Chemistry. 2014; 60: 838-847.

[22] Klinkenberg LJJ, van Dijk J, Tan FES, van Loon LJC, van Dieijen-Visser MP, Meex SJR. Circulating cardiac troponin T exhibits a diurnal rhythm. Journal of the American College of Cardiology. 2014; 63: 1788-1795.

[23] Gutenbrunner C. Circadian variations of the serum creatine kinase level-a masking effect? Chronobiology International. 2000; 17: 583-590.

[24] Klinkenberg LJJ, Wildi K, van der Linden N, Kouw IWK, Niens M, Twerenbold R, et al. Diurnal rhythm of cardiac troponin: consequences for the diagnosis of acute myocardial infarction. Clinical Chemistry. 2016; 62: 1602-1611.

[25] van der Linden N, Cornelis T, Klinkenberg LJJ, Kimenai DM, Hilderink JM, Litjens EJR, et al. Strong diurnal rhythm of troponin T, but not troponin $\mathrm{I}$, in a patient with renal dysfunction. International Journal of Cardiology. 2016; 221: 287-288.

[26] Nelson W, Tong YL, Lee JK, Halberg F. Methods for cosinorrhythmometry. Chronobiologia. 1979; 6: 305-323.

[27] Dubin RF, Li Y, He J, Jaar BG, Kallem R, Lash JP, et al. Predictors of high sensitivity cardiac troponin $\mathrm{T}$ in chronic kidney disease patients: a crosssectional study in the chronic renal insufficiency cohort (CRIC). BMC Nephrology. 2013; 14: 229.

[28] Reichlin T, Schindler C, Drexler B, Twerenbold R, Reiter M, Zellweger C, et al. One-hour rule-out and rule-in of acute myocardial infarction using high-sensitivity cardiac troponin T. Archives of Internal Medicine. 2012; 172: 1211-1218.

[29] Rubini Gimenez M, Twerenbold R, Jaeger C, Schindler C, Puelacher $\mathrm{C}$, Wildi $\mathrm{K}$, et al. One-hour rule-in and rule-out of acute myocardial infarction using high-sensitivity cardiac troponin I. American Journal of Medicine. 2015; 128: 861-870.

[30] Wildi K, Singeisen H, Twerenbold R, Badertscher P, Wussler D, Klinkenberg LJJ, et al. Circadian rhythm of cardiac troponin I and its clinical impact on the diagnostic accuracy for acute myocardial infarction. International Journal of Cardiology. 2018; 270: 14-20.

[31] Interventional Federation of Clinical Chemistry and Laboratory Medicine. High-Sensitivity* Cardiac Troponin I and $\mathrm{T}$ Assay Analytical Characteristics Designated by Manufacturer IFCC Committee on Clinical Applications of Cardiac Bio-Markers (C-CB). 2020. Available at: https://www.ifcc.org/media/478231/ high-sensitivity-cardiac-troponin-i-and-t-assayanalytical-characteristics-designated-by-manufacturerv122019.pdf (Accessed: 10 February 2021).
[32] Aschoff J. Circadian rhythms in man. Science. 1965; 148: 1427-1432.

[33] Doskin VA, Lavrent'eva NA. Circadian rhythms and their role in human physiology and pathology. Sovetskaia Meditsina. 1972; 35: 67-70. (In Russian)

[34] Sato M, Matsuo T, Atmore H, Akashi M. Possible contribution of chronobiology to cardiovascular health. Frontiers in Physiology. 2014; 4: 409.

[35] Panza JA, Epstein SE, Quyyumi AA. Circadian variation in vascular tone and its relation to $\alpha$-sympathetic vasoconstrictor activity. New England Journal of Medicine. 1991; 325: 986-990.

[36] Tofler GH, Brezinski D, Schafer AI, Czeisler CA, Rutherford JD, Willich $\mathrm{SN}$, et al. Concurrent morning increase in platelet aggregability and the risk of myocardial infarction and sudden cardiac death. New England Journal of Medicine. 1987; 316: 1514-1518.

[37] Fearnley GR, Balmforth G, Fearnley E. Evidence of a diurnal fibrinolytic rhythm; with a simple method of measuring natural fibrinolysis. Clinical Science. 1957; 16: 645-650.

[38] Suárez-Barrientos A, López-Romero P, Vivas D, Castro-Ferreira F, Núñez-Gil I, Franco E, et al. Circadian variations of infarct size in acute myocardial infarction. Heart. 2011; 97: 970-976.

[39] Arroyo Úcar E, Dominguez-Rodriguez A, Abreu-Gonzalez P. Influence of diurnal variation in the size of acute myocardial infarction. Medicina Intensiva. 2012; 36: 11-14. (In Russian)

[40] Seneviratna A, Lim GH, Devi A, Carvalho LP, Chua T, Koh T, et al. Circadian dependence of infarct size and acute heart failure in ST elevation myocardial infarction. PLoS ONE. 2015; 10: e0128526.

[41] Thornton SN. Overnight dehydration increases the risk of a morning infarct. Heart. 2011; 97: 1359.

[42] Manfredini R, Boari B, Bressan S, Gallerani M, Salmi R, Portaluppi F, et al. Influence of circadian rhythm on mortality after myocardial infarction: data from a prospective cohort of emergency calls. American Journal of Emergency Medicine. 2004; 22: 555-559.

[43] Ben Yedder N, Roux JF, Paredes FA. Troponin elevation in supraventricular tachycardia: primary dependence on heart rate. Canadian Journal of Cardiology. 2011; 27: 105-109.

How to cite this article: Aleksey Michailovich Chaulin, Dmitry V. Duplyakov. On the potential effect of circadian rhythms of cardiac troponins on the diagnosis of acute myocardial infarction. Signa Vitae. 2021;17(3):79-84. doi:10.22514/sv.2021.050. 